\title{
Improved detection of hepatic metastases of adrenocortical cancer by contrast-enhanced ultrasound
}

\author{
JUERGEN BAUDITZ $^{1 *}$, MARCUS QUINKLER $^{1 *}$, DIRK BEYERSDORFF $^{2}$ and WOLFRAM WERMKE ${ }^{1}$ \\ ${ }^{1}$ Internal Medicine, Center for Gastroenterology, Hepatology and Endocrinology; ${ }^{2}$ Department of \\ Radiology, Charité Campus Mitte, Charité University Medicine Berlin, Berlin, Germany
}

Received November 2, 2007; Accepted December 20, 2007

\begin{abstract}
Adrenocortical carcinoma (ACC) is a rare and heterogeneous malignancy whose pathogenesis and poor prognosis is poorly understood. Computerized tomography (CT) and magnetic resonance imaging (MRI) are routinely performed for the imaging of the adrenal mass and for standard staging of the chest and abdomen as the lung and liver are the primary organs for metastasis in ACC. Contrastenhanced ultrasound has been shown to have a high sensitivity and specifity for the differentiation of hepatic and neuroendocrine tumors. Twelve patients ( 7 women and 5 men; aged 24-74 years) with ACC were treated in our centre from 2004 to 2006 . The patients received staging with a contrast-enhanced multislice spiral computed tomography (MSCT) as well as with a conventional and an echoenhanced ultrasound of the liver. Contrast-enhanced ultrasound demonstrated liver metastases in 8 out of 12 patients $(67 \%)$ and MSCT in 6 out of 12 patients (50\%). In 2 out of 8 patients $(25 \%)$ MSCT did not detect the liver metastases. Even in retrospective analysis with knowledge of the ultrasound results, the hepatic lesions were not recognized by the MSCT, but became detectable by MSCT at a later time point. All hepatic lesions diagnosed by MSCT were also seen by ultrasound. The detection of liver metastases by ultra-sound resulted in a change of therapy in two patients. The contrast-enhanced ultrasound has a high sensitivity in detecting the highly-vascularized liver metastases of ACC and should be included in the staging algorithm of ACC.
\end{abstract}

Correspondence to: Dr Marcus Quinkler, Internal Medicine, Center for Gastroenterology, Hepatology and Endocrinology, Charité Campus Mitte, Charité University Medicine Berlin, Charitéplatz 1, D-10117 Berlin, Germany

E-mail:marcus.quinkler@charite.de

${ }^{*}$ Contributed equally

Key words: hepatic metastases, adrenocortical cancer, echoenhanced ultrasound

\section{Introduction}

Adrenocortical carcinoma (ACC) is a rare (1-2 cases per million and per year) and heterogeneous malignancy whose pathogenesis and poor prognosis is inadequately understood (1-3). Patients had adrenal steroid hormone excess (Cushing's syndrome, hyperandrogenemia, estrogen excess or hypermineralocorticolism) in $\sim 60 \%$ of cases (4). The size and appearance of an adrenal mass on computerized tomography (CT), magnetic resonance imaging (MRI), and more recently ${ }^{18} \mathrm{~F}$-fluorodeoxyglucose positron emission tomography (FDG-PET) have been used to differentiate between benign and malignant adrenal tumors. Surgery is the basis for treatment since the complete removal of a localized tumor is the best chance of cure (5). An incomplete resection of the primary tumor or metastatic disease not amenable to surgery is associated with a particularly poor prognosis. However, tumor debulking may help to control hormone excess and in individual cases opens up the possibility of other therapeutic options (3). There is, however, no medical treatment that has convincingly been shown to provide a definitive solution in locally invasive and/or metastatic ACCs thus far. Since the surgical removal of a local relapse or metastases is a valid therapeutic option, re-staging should be performed on a regular basis, e.g. every two to three months. CT and MRI are routinely performed for standard staging of the chest and abdomen as the lung and liver are the primary organs for metastasis in ACC. However, small metastases (diameter $<10 \mathrm{~mm}$ ) are often not visualized. Therefore we employed a high-resolution B-mode sonography and contrast-enhanced ultra-sound, two non-invasive procedures shown to have a high sensitivity and specifity for the differentiation of hepatic and neuroendocrine tumors (6-10), as an additional staging examination for liver metastases besides the contrastenhanced multislice computed tomography (MSCT) of the chest and abdomen in our 12 patients with ACC treated in our centre from 2004 to 2006 . Herein we report the outcome of hepatic staging by CT and contrast-enhanced ultrasound in our ACC patients.

\section{Patients and methods}

Patients. From September 2004 to September 2006 we treated 12 patients ( 7 women and 5 men) with histologicallyconfirmed adrenocortical cancer (ACC). The mean age was 
Table I. Characteristics of patients with ACC. ${ }^{a}$

\begin{tabular}{|c|c|c|c|c|c|c|c|}
\hline Sex & $\begin{array}{l}\text { Age at } \\
\text { diagnosis }\end{array}$ & $\begin{array}{l}\text { Tumor } \\
\text { size }(\mathrm{cm})\end{array}$ & Stage & $\begin{array}{l}\text { Hormone } \\
\text { production }\end{array}$ & $\begin{array}{l}\text { Survival } \\
\text { (months) }\end{array}$ & $\begin{array}{l}\text { Location of metastasis } \\
\text { during follow-up }\end{array}$ & Treatments \\
\hline $\mathrm{F}$ & 67 & 3 & 1 & None & $\begin{array}{c}19, \\
\text { Deceased }\end{array}$ & $\begin{array}{l}\text { Local recurrence, liver, } \\
\text { lung and spine }\end{array}$ & $\begin{array}{l}\text { Op, recurrence Op, } \\
\text { mitotane, EDP and Iressa } \\
\text { (ZD1839) }\end{array}$ \\
\hline $\mathrm{F}$ & 74 & 4.5 & 1 & Cortisol & 41 & $\begin{array}{l}\text { Local recurrence and } \\
\text { lung }\end{array}$ & $\begin{array}{l}\text { Op, recurrence Op and } \\
\text { mitotane }\end{array}$ \\
\hline M & 50 & 20 & 2 & None & 125 & None & Op and mitotane \\
\hline M & 60 & 12 & 2 & None & 77 & $\begin{array}{l}\text { Local recurrence, spleen } \\
\text { intestine and liver }\end{array}$ & $\begin{array}{l}\text { Op, recurrence Op, mitotane, } \\
\text { etoposid + carboplatin, } \\
\text { streptozosin and GT }\end{array}$ \\
\hline $\mathrm{F}$ & 41 & 8 & 2 & None & 18 & None & Op and mitotane \\
\hline $\mathrm{F}$ & 24 & 8 & 2 & Cortisol & 8 & Liver & Op, mitotane and EDP \\
\hline $\mathrm{F}$ & 68 & 13 & 3 & None & 53 & $\begin{array}{l}\text { Contralateral adrenal, } \\
\text { liver and para-aortal }\end{array}$ & $\begin{array}{l}\text { 2X Op, mitotane and } \\
\text { streptozosin }\end{array}$ \\
\hline M & 61 & 12 & 3 & None & 21 & Lung and liver & $\begin{array}{l}\text { Op, mitotane, EDP, } \\
\text { streptozosin, GT and RFA }\end{array}$ \\
\hline M & 44 & 13 & 4 & None & $\begin{array}{c}19, \\
\text { Deceased }\end{array}$ & $\begin{array}{l}\text { Local recurrence, lung, } \\
\text { liver, os sacrum and } \\
\text { skull }\end{array}$ & $\begin{array}{l}\text { Op, mitotane, EDP, } \\
\text { streptozosin and GT }\end{array}$ \\
\hline $\mathrm{F}$ & 58 & 12 & 4 & Cortisol + androgens & $\begin{array}{c}4, \\
\text { Deceased }\end{array}$ & $\begin{array}{l}\text { Local recurrence, } \\
\text { liver and lung }\end{array}$ & Op, mitotane and EDP \\
\hline $\mathrm{F}$ & 28 & 10 & 4 & Cortisol + androgens & $\begin{array}{c}25, \\
\text { Deceased }\end{array}$ & $\begin{array}{l}\text { Local recurrence, liver, } \\
\text { lung and rip }\end{array}$ & $\begin{array}{l}\text { Op, recurrence Op, mitotane, } \\
\text { EDP, streptozosin, GT and } \\
\text { RFA }\end{array}$ \\
\hline M & 59 & 5.5 & 4 & None & 8 & $\begin{array}{l}\text { Tumor thrombus } \\
\text { vena cava }\end{array}$ & Op, mitotane and radiation \\
\hline
\end{tabular}

${ }^{\text {aEDP }}=$ etoposide + cisplatin + doxorubicin; Op, operation; GT = gemcitabine + erlotinib and RFA, radiofrequency thermal ablation.

52.8 years (range: 24-74). According to the new Union International Contre Cancer (UICC) staging system published by the World Health Organisation (WHO) in 2004 (11), two patients had ACC stage 1, four patients stage 2, two patients stage 3 and four patients stage 4 at the time of diagnosis. The mean diameter of the ACC was $10.1 \mathrm{~cm}$ (range: 3-20). The patient characteristics are shown in Table I. All patients received surgical tumor removal. We performed a follow-up MSCT of the abdomen and chest every two months to monitor local recurrence or metastases. In addition, a percutaneous conventional high-resolution B-mode sonography and contrast-enhanced ultrasound were performed at the same time point as CT staging. For the contrast-enhanced ultrasound informed consent was obtained. The follow-up examination served as the gold standard for the presence of liver metastases. Patients without liver metastasis have been followed up for at least six month thus far.

Techniques. The sensitivity of the Doppler sonography for detecting low-blood flow velocity or small vessels has been considerably improved by the development of echo-enhancers, which consist of microbubbles. When exposed to an ultrasound field with a frequency of 1-5 MHz, microbubbles start to resonate and emit harmonic waves. First-generation echoenhancers such as Levovist ${ }^{\circledR}$ together with signal algorithms such as second harmonic imaging are capable of strongly enhancing the Doppler signal at 20-30 dB (12-14). However, these methods required a relatively high energy [high mechanical index, (MI)] for the resonation of micro-bubbles, resulting in a rapid microbubble destruction. The problem of microbubble instability has been solved by improved secondgeneration echo-enhancers such as sulphur hexafluoride (SonoVue ${ }^{\circledR}$ ) combined with a modified ultra-sound wave analysis (pulse inversion or, more recently, coded pulse sequences) (15). By using a low mechanical index (MI, 0.10.2 ) microbubbles can strongly resonate for longer time periods before being destroyed. The stability of the contrasting microbubbles is now sufficient to allow real-time sonography of the different hepatic vascularisation phases. Contrast-enhanced image analysis is possible for more than 
a
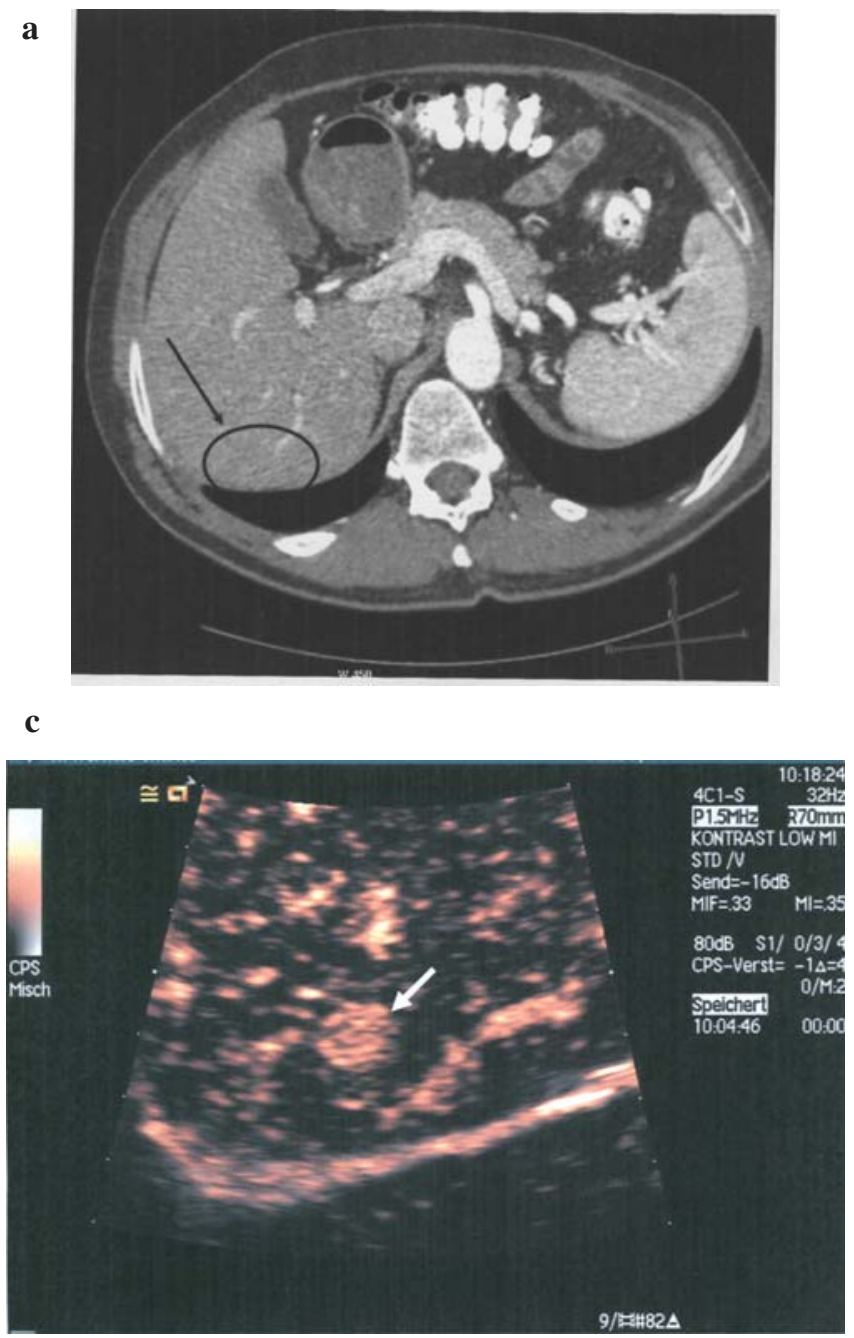

b

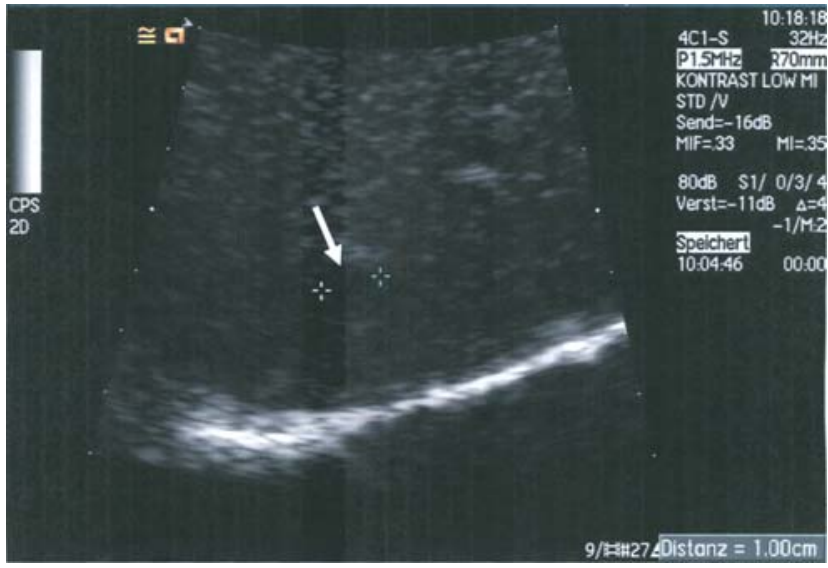

d

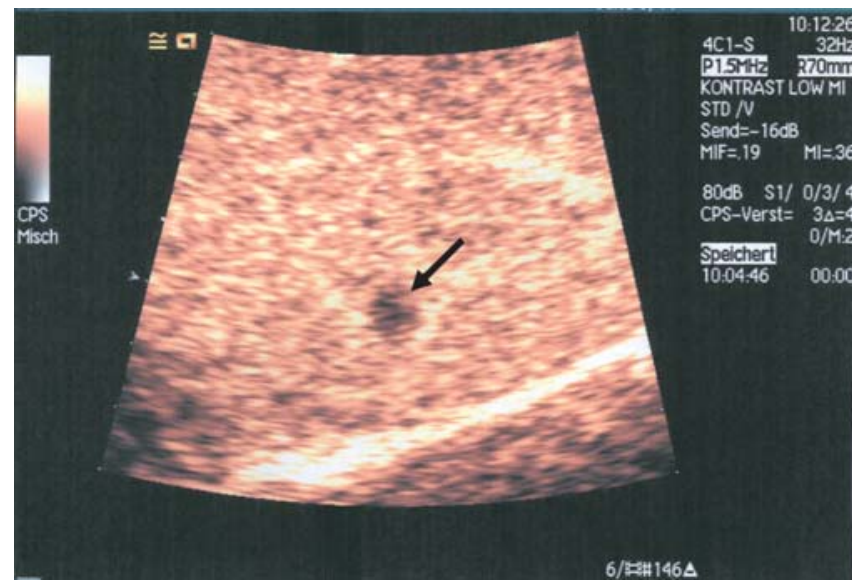

Figure 1. MSCT (a) of the suspected region of liver metastases (arrow and circle) in a 61-year-old patient with adrenocortical cancer. Visualization of a liver metastasis ( $1 \mathrm{~cm}$ in diameter) by B-mode sonography (b), by contrast-enhanced ultrasound showing early arterial phase (10 sec), (c) during the portalvenous phase (>30 sec) and (d) at the same time as MSCT

$5 \mathrm{~min}$. In contrast to the iodine-based computed tomography contrast agents, sulphur hexafluoride is completely non-critical in patients with renal insufficiency and hyperthyreodism and only very rarely causes allergic reactions. As there is no doselimiting toxicity, repeated injections are possible for a detailed analysis of the regions of interest. As with computed tomography, conventional B-mode sonography and contrastenhanced ultrasound should not be used as separate but supplemental imaging techniques (16).

All patients were investigated by an experienced examiner blinded to the patient laboratory and imaging findings. For the high-resolution B-mode sonography, a dynamic 2- to 5-Mhz sector scanner (HDI 5000/IU22, Philips) was used. After Bmode scanning, a contrast-enhanced ultrasound was performed with an Acuson Sequoia 512 (Siemens) and coded pulse sequences (CPS) with a low mechanical index (0.1-0.2). The image analysis started with a bolus injection $(0.025 \mathrm{ml} / \mathrm{kg}$ body weight) of the contrast agent SonoVue (Bracco International B.V., Amsterdam, The Netherlands) and lasted for 6-8 min. During the early and arterial-capillary phases (5-30 sec after injection) the architecture and intensity of arterial vascularisation was analysed and during the portal (30-120 sec) and late phases $(>120 \mathrm{sec})$ suspicious regions were investigated for the presence of physiological portal vessels and contrast filling.

Multislice spiral computed tomography was performed using a 16 Slice Scanner (MX 8000 IDT, Philips, Eindhoven, The Netherlands) with a slice of $1 \mathrm{~mm} 60 \mathrm{sec}$ after intravenous an administration of $120 \mathrm{ml}$ Ultravist 370 (Schering, Berlin, Germany) or $120 \mathrm{ml}$ Xenetix 350 (Gurbet, Paris, France) and an oral contrast with $50 \mathrm{ml}$ Peritrast oral. The images were evaluated by experienced readers blinded to the patient data.

\section{Results}

By using the B-mode sonography, the hepatic metastases of ACC appeared as echopoor or hypoechogenic and sharply delineated (Fig. 1b). After injecting the echo-enhancer, the liver metastases of ACC were found to be strongly hypervascularized. During the early arterial phase they rapidly (in the first $10 \mathrm{sec}$ after injection) enhanced (Fig. 1c) before the filling of the normal liver parenchyma. When the portalvenous system filled with the echo-enchancer (portal phase, 30-90 sec 
after injection) the metastases had already washed out the contrast enhancer from the arterial phase and (since no portalvenous vessels are present in malignant tissue) were visible as a sharp contrast defect (Fig. 1d).

Multislice spiral computed tomography (MSCT) detected liver metastases in 6 out of 12 patients $(50 \%)$, but echoenhanced ultrasound demonstrated liver metastases in 8 out of 12 patients $(67 \%)$. Liver metastases were not detected in 2 out of 8 patients $(25 \%)$ by MSCT, even retrospectively and with knowledge of the ultrasound results (a 61-year-old male with stage 3 ACC and a 44-year-old male with stage 4 ACC at diagnosis). In one case, an additionally performed contrastenhanced biphasic CT scan failed to detect the liver metastasis. However, at a new re-staging two and four months later, MSCT detected the liver metastases seen in the echoenhanced ultrasound at an earlier stage. All hepatic lesions diagnosed by MSCT were also seen by echo-enhanced ultrasound. The detection of liver metastases by echoenhanced ultrasound resulted in a change of the therapy in the two patients. They have been on a regimen of etoposide, doxorubicin and cisplatin combined with mitotane due to their lung metastases, and were changed to streptozosin plus mitotane due to these newly-diagnosed hepatic metastases. In the follow-up, one patient successfully received RFA (radiofrequency thermal ablation) of his three liver lesions after the diagnosis with the contrast-enhanced ultrasound.

\section{Discussion}

Imaging is important not only for characterizing adrenal tumors but also for staging in ACC. Since the surgical removal of a local relapse or metastases is a valid therapeutic option, re-staging should be performed on a regular basis, e.g. every two to three months. Surgical tumor debulking may help to control hormone excess and in individual cases opens up the possibility of other therapeutic options (3). CT and MRI are routinely performed for standard staging of the chest and abdomen as the lung and liver are the primary organs for metastasis in ACC. Unfortunately, small metastases (diameter $<10 \mathrm{~mm}$ ) are often not visualized. However, it is important to detect the metastases of ACC at an early stage to choose the optimal therapeutic strategy (e.g. surgical resection, radiofrequency thermal ablation therapy, systemic chemotherapy and mitotane treatment). Therefore, a non-invasive and costefficient method with high diagnostic accuracy is required, which detects hepatic metastases at an early stage.

The hypervascularization of liver ACC metastases may be the reason why they were not detected by the MSCT. Contrastenhanced CT is performed $60 \mathrm{sec}$ after starting the intravenous injection. However, this seems to be too late to demonstrate the arterial filling phase of the hepatic ACC lesion, which occurs within a few seconds as shown by the echo-enhanced ultrasound. During the portal phase, small lesions of $<1 \mathrm{~cm}$ may not be detected by CT if the wash-out of the contrast agent is still incomplete. A multiphase MSCT with shorter acquisition intervals would probably detect the metastases, but is not performed due to the high radiation exposure. MRI can detect liver metastases, but is not routinely performed, because of limited capacities and higher costs.
In our patient cohort, liver metastases were not detected in 2 out of 8 patients by MSCT. The hepatic metastases of the two patients were small lesions (diameter $<12 \mathrm{~mm}$ ) and were able to be seen by conventional ultrasound and echo-enhanced sonography. The hepatic lesions, seen in the subsequent follow-up staging with MSCT two and four months later, had been increasing in size throughout the following staging procedures. Even retrospectively and with knowledge of the ultrasound results, the hepatic lesions were not recognized by MSCT at the time point when they were diagnosed by echoenhanced ultrasound. It is assumed that MSCT is a sensitive method capable of detecting all liver metastases. However, smaller hypervascularized liver metastases of ACC seem to escape MSCT detection. In this small series of ACC patients, we have shown that a conventional ultrasound combined with echo-enhanced ultrasound is a sensitive and useful method of increasing the sensitivity of the staging process in ACC. The advantage of the contrast-enhanced ultrasound is important in early the stages of ACC when there is a question of instituting early treatment versus observation after the primary lesion has been surgically removed and there is no evidence of metastases. However, when there are multiple hepatic and/or pulmonary metastatic lesions, detecting merely a few additional lesions will probably not always affect overall management.

In conclusion, the contrast-enhanced ultrasound has a high sensitivity in detecting highly-vascularized liver metastases of ACC and should be included in the staging algorithm of ACC at least when no liver metastases are detected by CT. This may offer the possibility of an earlier therapeutic intervention either surgically, with radiofrequency thermal ablation or with systemic therapy. However, to what extent this procedure could change the overall outcome of the disease remains uncertain.

\section{References}

1. Wajchenberg BL, Albergaria Pereira MA, Medonca BB, et al: Adrenocortical carcinoma: Clinical and laboratory observations. Cancer 88: 711-736, 2000.

2. Abiven G, Coste J, Groussin L, et al: Clinical and biological features in the prognosis of adrenocortical cancer: Poor outcome of cortisol-secreting tumors in a series of 202 consecutive patients. J Clin Endocrinol Metab 91: 2650-2655, 2006.

3. Allolio B and Fassnacht M: Clinical review: Adrenocortical carcinoma: clinical update. J Clin Endocrinol Metab 91: 2027-2037: 2006.

4. Koschker AC, Fassnacht M, Hahner S, Weismann D and Allolio B: Adrenocortical carcinoma - improving patient care by establishing new structures. Exp Clin Endocrinol Diabetes 114: 45-51, 2006.

5. Schteingart DE, Doherty GM, Gauger PG, et al: Management of patients with adrenal cancer: Recommendations of an international consensus conference. Endocr Relat Cancer 12: 667-680, 2005.

6. Fracanzani AL, Burdick L, Borzio M, et al: Contrast-enhanced Doppler ultrasonography in the diagnosis of hepatocellular carcinoma and premalignant lesions in patients with cirrhosis. Hepatology 34: 1109-1112, 2001.

7. Rickes S, Ocran K, Schulze S and Wermke W: Evaluation of Doppler sonographic criteria for the differentiation of hepatocellular carcinomas and regenerative nodules in patients with liver cirrhosis. Ultraschall Med 23: 83-90, 2002.

8. Beissert M, Delorme S, Mutze S, et al: Comparison of B-mode and conventional colour/power Doppler ultrasound, contrastenhanced Doppler ultrasound and spiral CT in the diagnosis of focal lesions of the liver: Results of a multicentre study. Ultraschall Med 23: 245-250, 2002. 
9. Rickes S, Schulze S, Neye H, Ocran KW and Wermke W: Improved diagnosing of small hepatocellular carcinomas by echo-enhanced power Doppler sonography in patients with cirrhosis. Eur J Gastroenterol Hepatol 15: 893-900, 2003.

10. Rickes S, Ocran KW, Gerstenhauer G, Neye H and Wermke W: Evaluation of diagnostic criteria for liver metastases of adenocarcinomas and neuroendocrine tumours at conventional ultrasound, unenhanced power Doppler sonography and echoenhanced ultrasound. Dig Dis 22: 81-86, 2004.

11. DeLellis R, Lloyd RV, Heitz PU and Eng C: World Health Organization classification of tumours. In: Pathology and Genetics of Tumours of Endocrine Organs. IARC Press, Lyon, 2004.

12. Calliada F, Campani R, Bottinelli O, Bozzini A and Sommaruga MG: Ultrasound contrast agents: basic principles. Eur J Radiol 27: S157-S160, 1998.
13. Correas JM, Helenon O, Pourcelot L and Moreau JF: Ultrasound contrast agents. Examples of blood pool agents. Acta Radiol 412: 101-112, 1997

14. Wermke W and Gassmann B: Tumor Diagnostics of the Liver with Echo Enhancers. Springer, Berlin, New York, 1998.

15. Kim AY, Choi BI, Kim TK, Kim KW, Lee JY and Han JK: Comparison of contrast-enhanced fundamental imaging, second-harmonic imaging, and pulse-inversion harmonic imaging. Invest Radiol 36: 582-588, 2001.

16. Wermke W: Sonographische Differenzialdiagnose Leberkrankheiten. Deutscher Ärzteverlag, Cologne, 2005. 